\title{
Otological Hemangioma: A Case Report and Literature Review
}

\author{
${ }^{1}$ Alok Rathi, ${ }^{2}$ Kamran A Syed, ${ }^{3}$ Mayur Suryawanshi, ${ }^{4}$ Mary John
}

\section{ABSTRACT}

Aim: To report a rare case of hemangioma involving external and middle ear.

Background: Vascular anomalies are common in head and neck but rarely reported in the ear. Hemangiomas are tumors growing with age. They can cause significant morbidity in the enclosed spaces of the ear.

Case report: A 6-year-old boy presented with itching and pain in the right ear on evaluation had a polyp in the external auditory canal. He underwent excision biopsy of the lesion which was reported as hemangioma involving external and middle ear.

Conclusion: Otological hemangioma is not much studied. We here report a case of hemangioma involving both external and middle ear, which is sixth such case in the literature.

Clinical significance: Knowing the various presentations will help in early diagnosis and appropriate management of these lesions

Keywords: External ear, Hemangioma, Mastoid, Middle ear.

How to cite this article: Rathi A, Syed KA, Suryawanshi M, John M. Otological Hemangioma: A Case Report and Literature Review. Int J Otorhinolaryngol Clin 2017;9(3):102-105.

Source of support: Nil

Conflict of interest: None

\section{BACKGROUND}

Vascular anomalies are the most common congenital and neonatal dysmorphogenesis affecting children with $60 \%$ being located in the head and neck. ${ }^{1}$ They are classified into vascular tumors and vascular malformations. ${ }^{2}$ Hemangioma is the most common tumor of infancy and childhood with the prevalence of $4.5 \%$ in this age group. However, there are other vascular malformations which

${ }^{1}$ Fellow, ${ }^{2,3}$ Assistant Professor, ${ }^{4}$ Professor

${ }^{1}$ Department of Otolaryngology Head and Neck Surgery, Christian Medical College, Vellore, Tamil Nadu, India

2,4 Department of ENT, Christian Medical College, Vellore, Tamil Nadu, India

${ }^{3}$ Department of Histopathology, Christian Medical College, Vellore, Tamil Nadu, India

Corresponding Author: Alok Rathi, Fellow, Department of Otolaryngology Head and Neck Surgery, Christian Medical College, Vellore, Tamil Nadu, India, e-mail: alokrathi14@gmail. com may present in adulthood. ${ }^{1}$ Their behavior in the ear is not much known due to its rarity at this site. We here report a case of external and middle ear hemangioma, sixth such in English literature and critically review the literature of otological hemangiomas.

\section{CASE DESCRIPTION}

A 6-year-old boy presented to pediatric otolaryngology outpatient department with complaints of occasional pain and itching in right ear for past 6 months. There was an associated history of hearing loss with no history of ear discharge or bleeding. Examination revealed a pale greyish polyp filing external auditory canal, insensitive to touch. The tympanic membrane could not be visualised. High resolution computed tomography showed homogenous soft tissue density filling external auditory canal, middle ear and mastoid antrum with intact ossicular chain. These radiological features were suggestive of chronic otitis media with an aural polyp (Graph 1 and Fig. 1). The child underwent mastoid exploration to reveal bleeding mass filling external auditory canal, mastoid antrum and mesotympanum engulfing the ossicular chain (Fig. 2). Mass was cleared preserving the ossicular chain.

Histopathological examination showed polypoidal growth with lobular configuration composed of closely packed capillary-sized vascular spaces without any granulomas (Fig. 3). The CD34 stain was positive, conforming with the diagnosis of lobular capillary hemangioma (LCH). The child has been asymptomatic after surgery and on follow-up for 4 months.

\section{DISCUSSION}

The terminology of vascular anomalies had been very confusing with term hemangioma often used to describe various vascular anomalies in the literature. Efforts of International Society for Study of Vascular Anomalies (ISSVA) to classify these lesions based on their constitution, natural course, and treatment has led to the current classification. ${ }^{2}$ The importance of accurate nomenclature lies in appropriate management of these entities.

A literature search showed 53 case reports of otological hemangiomas (Table 1). Most common lesions being cavernous and capillary hemangiomas, but both belong to different groups with contrasting 

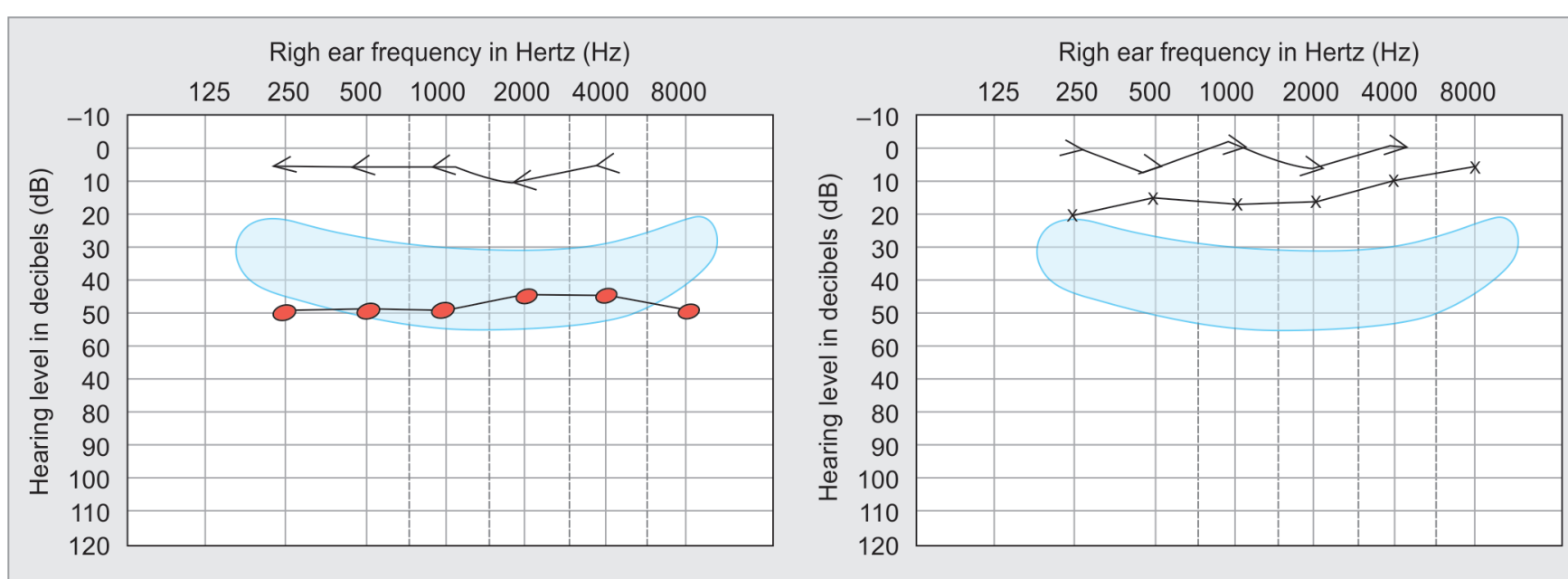

Graph 1: Pure tone audiogram showing moderate conductive hearing loss in right ear

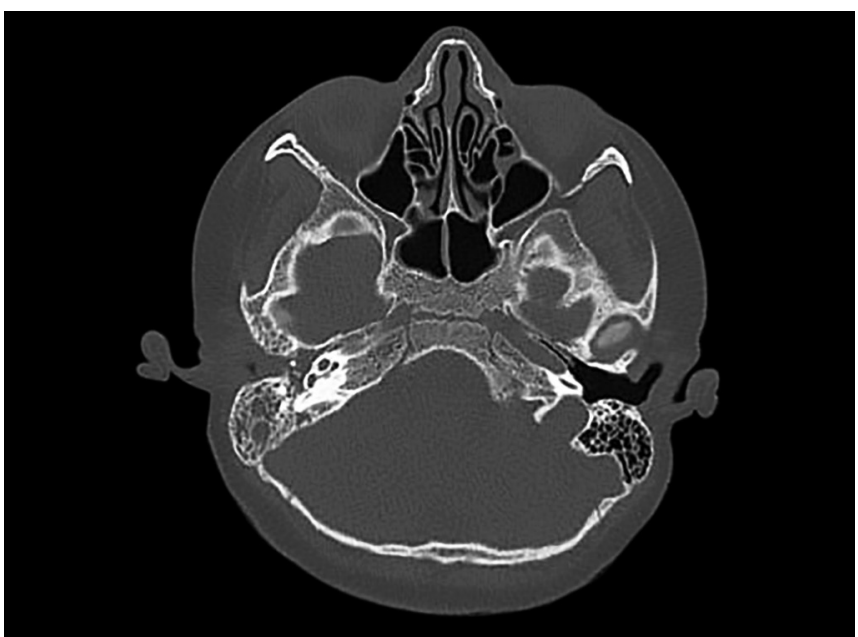

Fig. 1: High resolution computed tomography axial section showing homogenous soft tissue density involving mastoid, middle ear and external ear

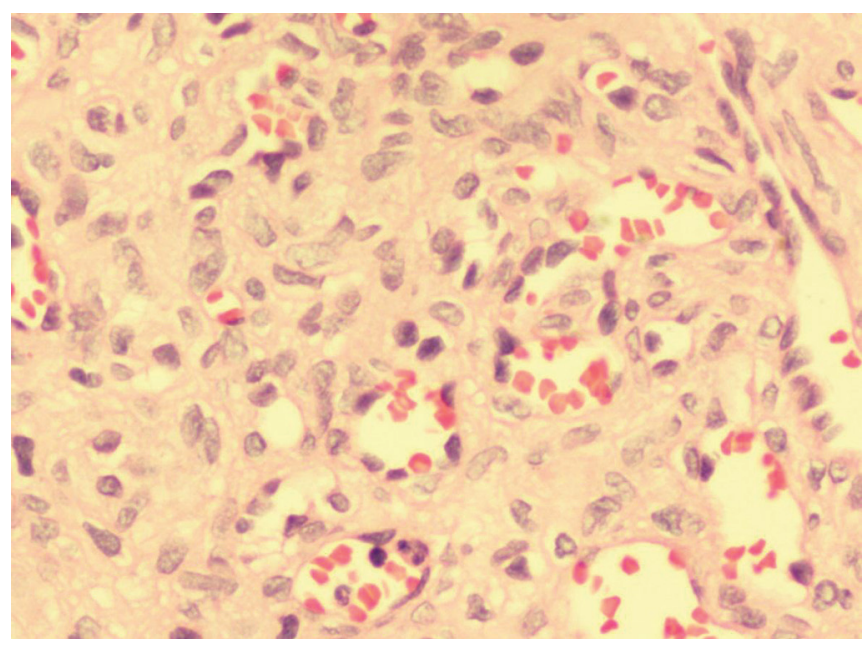

Fig. 3: Histopathological staining of specimen showing lobules of closey packed endothelial lined capillaries conforming diagnosis of hemangioma

management. Capillary hemangioma is classified as a vascular tumor while cavernous lesions are vascular malformations. ${ }^{1,2}$ Analysis of these articles suggest that the management of otological lesions depends

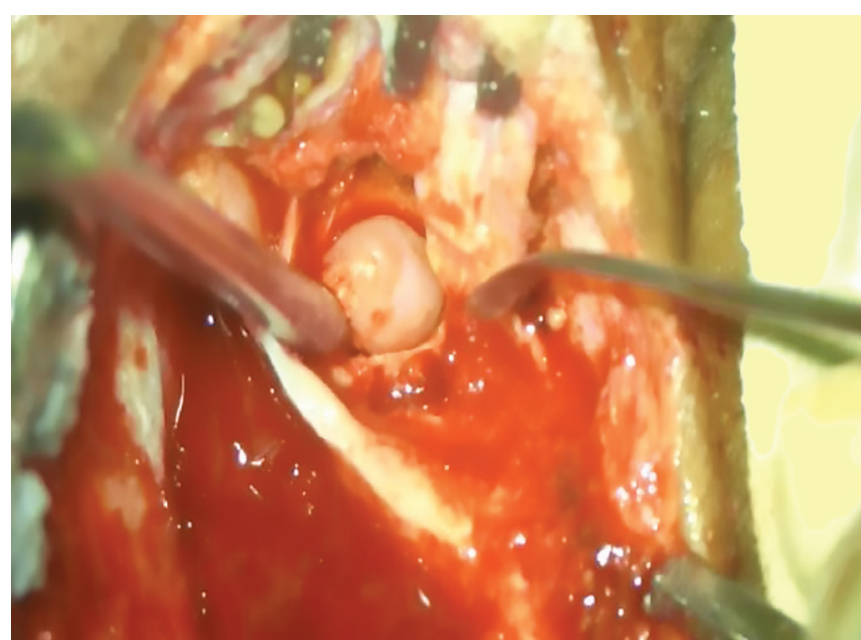

Fig. 2: Pale pinkish polyp filling external auditory canal

Table 1: Otological hemangiomas reported after 1982

\begin{tabular}{lllll}
\hline & Capillary & Cavernous & Hemangioma & Total \\
\hline EAC +/- TM & 7 & 14 & 8 & 29 \\
$\begin{array}{l}\text { Tympano- } \\
\text { mastoid }\end{array}$ & 13 & 2 & 4 & 19 \\
Combined & 2 & 3 & 0 & \\
Total & 22 & 19 & 12 & 53 \\
\hline
\end{tabular}

EAC: External auditory canal; TM: Tympanic membrane

primarily on presentation and diagnosis. They can be broadly grouped as follows:

- External ear group

- Middle ear group

- Combined group

\section{External Ear Group}

These include lesions involving either external auditory canal (EAC) alone or tympanic membrane (TM) alone or both. These lesions are seen predominantly in adult males with the majority being a cavernous hemangioma. ${ }^{3}$ Majority of these arise from lamina propria of posterior half of TM and may have extensions to adjacent bony EAC 
or other parts of TM. They may be incidentally detected or present with symptoms of otitis media, hearing loss, tinnitus, foreign body sensation or ear bleed. ${ }^{4}$ The examination often reveals a reddish to purple vascular mass which is usually seen over TM and EAC. Sometimes mass may be completely occluding EAC obstructing the view of the TM. ${ }^{3,4}$

\section{Middle Ear Group: Further Divided into Two Subtypes}

- Hemangioma related to facial canal

- Tympanomastoid hemangioma

Facial canal hemangioma: These form the most common hemangioma of the middle ear with the site of origin in decreasing order of frequency-perigeniculate ganglion, internal auditory canal and at the origin of chorda tympani. ${ }^{5}$ The close relationship and communications of vessels at these sites explain the high incidence. They typically present with recurrent gradually progressive facial palsy. They are more symptomatic with respect to the small size of lesion and require treatment as early as possible. ${ }^{5}$

Tympanomastoid hemangioma: These include lesions involving the middle ear (ME) alone, $\mathrm{ME} / \mathrm{mastoid}, \mathrm{ME} /$ TM. These lesions are seen more common in adults (15 vs. 4) with capillary variant seen more than cavernous (Table 2). They usually present with hearing loss or other symptoms of tinnitus, dizziness, otorrhoea and are rarely detected incidentally. ${ }^{6}$ Otoscopy shows reddish mass behind TM, more at the posterior half and attic than at the anterior half. ${ }^{7,8}$ Glomus, being the most common benign tumor of middle ear, is the usual clinical diagnosis.
Radiological investigations are indicated to study the site of origin, extent, and type of lesion. Ossicles are usually intact, but often engulfed by the lesion, and are rarely eroded. ${ }^{9,10}$ Unusually, tegmen was eroded in one case, and facial palsy was noted in the other. ${ }^{11,12}$

\section{Combined Group}

These lesions involve both the external and middle ear. They may also extend beyond mastoid to involve the skin, occiput, and other adjacent areas. Only five cases are reported in English literature till date. All the reports but one are in the adults, symptoms including hearing loss, tinnitus, bleeding or as otitis media. ${ }^{7}$ Clinically reddish polypoidal mass is seen in EAC occluding further visualization. ${ }^{13}$ Imaging is indicated to classify and assess the extent of the lesion. ${ }^{14}$ Bony structures are usually intact, however erosion of EAC, ossicles and facial canal are reported in these extensive entities. ${ }^{13,14}$

\section{CONCLUSION}

Hemangioma of the ear is being reported more frequently with increasing clarity of classification and behavior of vascular anomalies. Their management depends on the diagnosis and its presentation. Surgery is the treatment of choice with complete excision. Further studies are needed to understand the chance of recurrence.

\section{CLINICAL SIGNIFICANCE}

Polyp in EAC has huge differential diagnosis, requiring surgical intervention in most of the cases. Knowing various presentations of vascular lesions in ear including

Table 2: Tympanomastoid hemangioma

\begin{tabular}{llllll}
\hline Author & Age & Sex & Location & Symptom & Pathology \\
\hline Wuyts, 2014 & 58 & M & Tympanomastoid & Vertigo, tinnitus & Capillary \\
Karatas, 2012 & 62 & F & Promontory & Otorrhoea, tinnitus, HL & Capillary \\
Nouri, 2012 & 60 & F & ET, MT & HL & Capillary \\
Bittencourt, 2012 & 35 & F & Posterior MT & HL, tinnitus & Hemangioma \\
Pistorio, 2011 & 77 & M & Tympanomastoid & Otorrhagia, HL, aural fullness & Capillary \\
Kojima, 2008 & 51 & M & Tympanomastoid & HL, aural fullness & Capillary hemangioma \\
Hsueh, 2007 & 56 & F & Promontory & Tinnitus & Capillary \\
Davids, 2006 & NA & NA & MT & NA & Capillary \\
Fierek, 2004 & 6 & M & Tympanomastoid & Recurrent otitis media & Capillary \\
Tokyol, 2003 & 51 & F & Anterior MT & Tinnitus, vertigo & Capillary \\
Hetch, 2001 & 4 & F & Anterior MT & Otitis media & Capillary \\
Payman, 1999 & $4 / 12$ & M & ET, MT & Asymptomatic & Capillary \\
Palacios, 1999 & NA & NA & Temporal bone & Facial palsy & Hemangioma \\
Manning, 1999 & & M & Bilateral MT & HL, tinnitus & Capillary \\
Teknos, 1997 & 80 & F & ET, superior MT & HL & Hemangioma \\
Mair, 1994 & 29 & M & Posterior MT & Asymptomatic & Cavernous hemangioma \\
Rutka, 1989 & NA & NA & Incus & Incidental & Cavernous \\
Jackson, 1987 & 1.1 & M & Posterior MT & Otitis media & Hemangioma \\
Dayal, 1982 & 64 & F & Posterior MT & HL & Capillary \\
\hline
\end{tabular}

ET: Epitympanum; MT: Mesotympanum; HL: Hearing loss 
polyp in EAC helps in early diagnosis and treatment of underlying disease. These are the separate group of lesions with increasing understanding of their biological behavior in the recent past. With new treatment options developing rapidly, surgical intervention may be only for diagnosis in the near future.

\section{REFERENCES}

1. Buckmiller LM, Richter GT, Suen JY. Diagnosis and management of hemangiomas and vascular malformations of the head and neck. Oral Dis 2010;16(5):405-418

2. Wassef M, Blei F, Adams D, et al. Vascular anomalies classification: recommendations from the International Society for the Study of Vascular Anomalies. Pediatrics 2015;136(1):e203-14

3. Mevio E, Cazzaniga M, Mullace M, et al. Hemangioma of the Tympanic membrane: a case and a review of the literature. Case Reports in Otolaryngology 2012;2012:402630.

4. Yamamoto K, Ogasawara N, Seki N, et al. Vascular tumors of the external auditory canal: three case reports and a review of the literature. Springer Plus 2015;4:307.

5. Semaan MT, Slattery WH, Brackmann DE. Geniculate ganglion hemangiomas: clinical results and long-term follow-up. Otol Neurotol 2010;31:665-670

6. Nouri H, Harkani A, Elouali Idrissi M, et al. Capillary Hemangioma of the Middle Ear: One Case Report and
Review of the Literature. Case Reports in Otolaryngology. 2012;2012:305172.

7. Hecht DA, Jackson CG, Grundfast KM. Management of middle ear hemangiomas. Am J Otolaryngol 2001; 22(5):362-366.

8. Tokyol C, Yilmaz MD. Middle ear hemangioma: a case report. Am J Otolaryngol 2003;24(6):405-407.

9. Payman RN, Mortelliti AJ, Gacek RR, et al. Middle ear capillary hemangioma in an infant. Am J Otolaryngol 1999;20(1):59-63.

10. Fierek O, Laskawi R, Kunze E. Large intraosseous hemangioma of the temporal bone in a child. Ann Otol Rhinol Laryngol 2004;113(5):394-398.

11. Wuyts L, Potvin J, Vanderveken OM, et al. Middle ear capillary haemangioma causing vestibulocochlear symptoms: a case report. B-ENT 2014;10(3):237-243.

12. Palacios E, Valvassori G. Temporal bone hemangioma as a cause of facial paralysis. Ear Nose Throat J 1999;78(2): 84 .

13. Odat H, Al-Qudah M, Al-Qudah MA. Cavernous hemangioma of the external canal, tympanic membrane, and middle ear cleft: a case report. Head Neck Pathol. 2015 Aug 25.

14. Pavamani SP, Surendrababu NR, Ram TS, et al. Capillary haemangioma involving the middle and external ear: radiotherapy as a treatment method. Australas Radiol 2007;51(4):394-397. 